\title{
Unanticipated Ruptured Sinus of Valsalva Aneurysm in a Patient with Subpulmonic Ventricular Septum Defect: Suspected by Intraoperative Transthoracic Echo; Confirmed by Intraoperative Transesophageal Echo
}

\author{
${ }^{1}$ Imran Hussain Bhat, ${ }^{2}$ Ravi Raj, ${ }^{3}$ Goverdhan Dutt Puri
}

\begin{abstract}
A sinus of valsalva (SOV) aneurysm is a rare cardiac anomaly that may be congenital or acquired and may be associated with other cardiac lesions. If the aneurysm ruptures, it causes acute symptoms of dyspnea. Echocardiography is a useful diagnostic tool that can guide in proper management of these patients. We present a case of subpulmonic ventricular septal defect (VSD) in which ruptured SOV was detected by intraoperative transthoracic echocardiography and confirmed by transesophageal echocardiography (TEE) which helped in adequate surgical repair and good outcome for the patient. This case report emphasizes the importance of routine transthoracic and TEE in operating room by cardiac anesthesiologist to confirm the original diagnosis and look for new unanticipated findings, especially in a patient with strong clinical suspicion.
\end{abstract}

Keywords: Ruptured sinus of valsalva aneurysm, Subpulmonic ventricular septum defect, Transesophageal echocardiography, Transthoracic echocardiography.

How to cite this article: Bhat IH, Raj R, Puri GD. Unanticipated Ruptured Sinus of Valsalva Aneurysm in a Patient with Subpulmonic Ventricular Septum Defect: Suspected by Intraoperative Transthoracic Echo; Confirmed by Intraoperative Transesophageal Echo. J Perioper Echocardiogr 2015;3(1): 25-28.

\section{Source of support: Nil}

Conflict of interest: None

\section{INTRODUCTION}

Sinus of valsalva (SOV) aneurysm is an uncommon disease with incidence ranging from 0.1 to $3.5 \%$ and male to female predominance of 4:1, with the highest incidence in the Asian population. ${ }^{1}$ It is commonly associated

\footnotetext{
${ }^{1,2}$ Senior Resident, ${ }^{3}$ Professor

${ }^{1-3}$ Department of Anesthesia and Intensive Care, Postgraduate Institute of Medical Education and Research, Chandigarh, India
}

Corresponding Author: Ravi Raj, Senior Resident, Department of Anesthesia and Intensive Care, Postgraduate Institute of Medical Education and Research, Chandigarh, India, Phone: 01722755065, e-mail: drraviraj2007@gmail.com with ventricular septal defect (VSD), aortic stenosis and bicuspid aortic valve. The mean age of presentation for SOV is 35 years (13-65 years), whereas rupture sinus of valsalva (RSOV) aneurysm often occurs after puberty but before 40 years and causes acute clinical deterioration of the patient. ${ }^{2}$ Rupture sinus of valsalva involves most commonly right coronary sinus (75\%), followed by noncoronary cusp $(20 \%)$ and rarely involves left coronary sinus. ${ }^{3}$ Typically, SOV aneurysm most frequently ruptures into the right ventricle (around $60 \%$ ), less frequently right atrium (approximately $30 \%$ ) and infrequently into the left atrium, left ventricle or pericardium. ${ }^{4}$

Morphologically, SOV aneurysms are thin-walled tubular or saccular outpouchings from aortic sinuses and occurs due to structural weakness of the aortic media, leading to subsequent avulsion and aneurysm formation. Congenitally, there may be incomplete fusion of the right and left distal bulbous septum, the base of which forms the right and the noncoronary sinuses of valsalva. Acquired causes often involve severe infections with syphilis and or endocarditis, trauma, severe atherosclerosis and aortic dissection that alter the anatomy and structural integrity of the sinuses leading to aneurysm formation. ${ }^{5}$

Transthoracic echocardiography (TTE) is the first-line investigation which shows aneurysmal tissue of SOV protruding into right heart most commonly and some times left heart chambers. Color Doppler interrogation shows blood flow/abnormal communication in case it has ruptured. Transesophageal echocardiography (TEE) is more sensitive and specific for pathologies involving aortic valve or aortic root including RSOV. We present a case of subpulmonic ventricular septum defect, in which an unanticipated finding of RSOV was initially detected by intraoperative TTE and further confirmed by TEE.

\section{CASE REPORT}

A 39-year-old female presented with 1 month history of progressively increasing dyspnea and generalized weakness. She had no significant medical or surgical history. On physical examination, she was tachypneic 


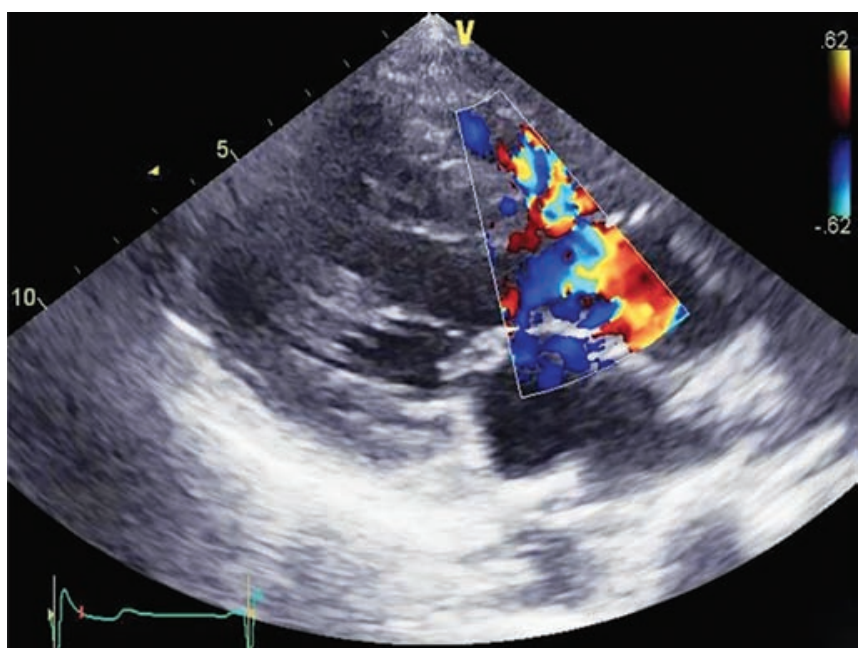

Fig. 1: Parasternal long axis view showing color flow across interventricular septum consistent with a ventricular septum defect

and her vitals included heart rate of $124 /$ minute and blood pressure 132/42 mmHg. Preoperative lab inveastigations showed hemoglobin of $7.4 \mathrm{~g} / \mathrm{dl}$, normal platelet count, leukocyte count and normal renal and liver function test. Her chest X-ray and electrocardiogram (ECG) were also normal. Her preoperative TTE report mentioned a perimembranous VSD measuring $12 \mathrm{~mm}$, a subaortic membrane with peak left ventricular outflow tract gradient (68 $\mathrm{mm} \mathrm{Hg}$ ), mild aortic regurgitation, mild mitral regurgitation and normal left ventricular systolic function. The patient was scheduled for VSD closure and resection of subaortic membrane.

Inside the operating room, standard American Society Anaesthesiolgists (ASA) monitoring was established, a left radial arterial line was placed, right internal jugular vein was cannulated with a 7 French, triple lumen catheter, and patient's airway was secured with a $7 \mathrm{~mm}$ endotracheal tube after induction of general anesthesia. Initial TTE examination by cardiac anesthesiologist intraoperatively

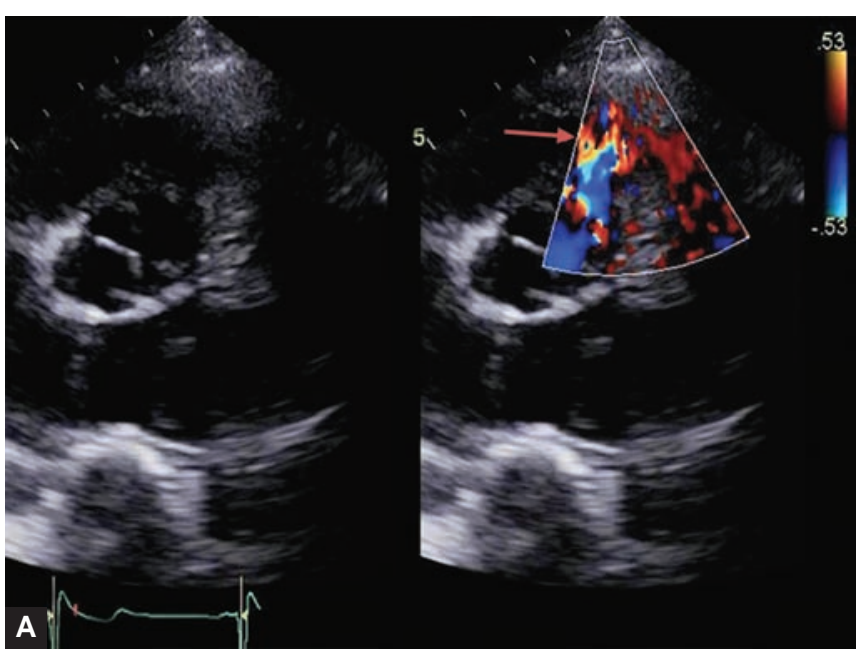

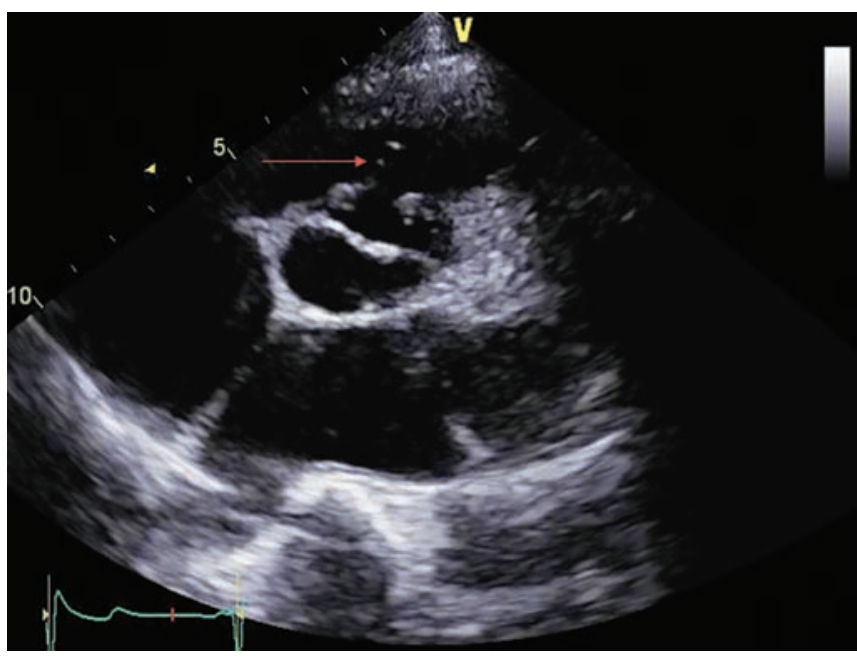

Fig. 2: Parasternal short axis view showing aneurysmal tissue (arrow) prolapsing into right ventricular outflow tract

showed a color flow jet from left to right ventricle in parasternal long axis (Fig. 1, Video 1) consistent with a VSD. However, parasternal short axis view revealed a subpulmonic VSD and aneurysmal tissue just above right coronary cusp protruding into right ventricular outflow tract region (Fig. 2, Video 2) and two color flow jets in right ventricular outflow tract; one an early systolic jet consistent with subpulmonic VSD (Fig. 3A, Video 3) and another a late/end systolic jet continuing in diastole, consistent with ruptured sinus of valsalva aneurysm (Fig. 3B, Video 3). Transesophageal echocardiography examination (6VT-D, GE vivid E9; GE Vingmed Ultrasound AS, Horten, Norway) in midesophageal aortic valve long axis view revealed aneurysmal tissue prolapsing into right ventricular outflow tract (RVOT) (Fig. 4, Video 4). Color flow Doppler interrogation over the same region showed two jets; one early systolic jet due to a subpulmonic VSD (Fig. 5, Video 5) and another mid/ late systolic jet due to a ruptured SOV aneurysm (Fig. 6).

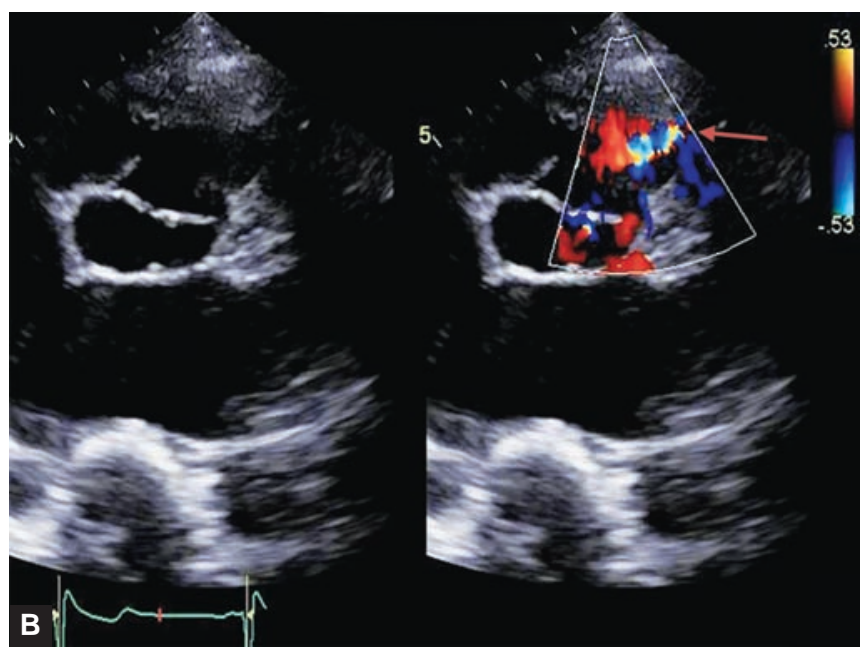

Figs 3A and B: (A) Parasternal short axis view showing early systolic flow (arrow) consistent with a subpulmonic ventricular septal defect and (B) Parasternal short axis view showing late systolic flow (arrow) in the right ventricular outflow tract region consistent with ruptured sinus of valsalva aneurysm 


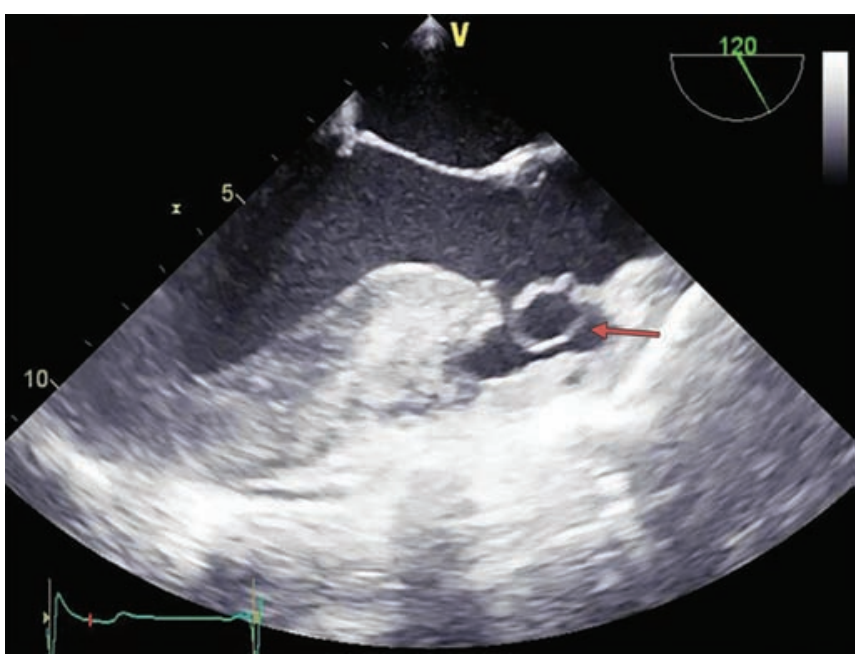

Fig. 4: Midesophageal aortic valve long axis view showing aneurysmal tissue (arrow) prolapsing into right ventricular outflow tract region

There was no evidence of any subaortic membrane/left ventricular outflow tract obstruction on TTE and TEE. Other findings included mild aortic regurgitation and normal biventricular function.

The above findings were conveyed to the surgical team. The surgical team confirmed the above-mentioned echocardiography findings and the patient underwent polytetrafluoroethylene (PTFE) patch closure of VSD and repair of RSOV via RVOT approach on cardiopulmonary bypass. There was no residual defect on repeat TEE examination after coming off bypass.

\section{DISCUSSION}

There was an unanticipated new finding of RSOV on intraoperative TTE which was confirmed by TEE. The presence of characteristic windsock deformity of right coronary sinus and presence of two differently directed color jet flows on parasternal short axis view (TTE)

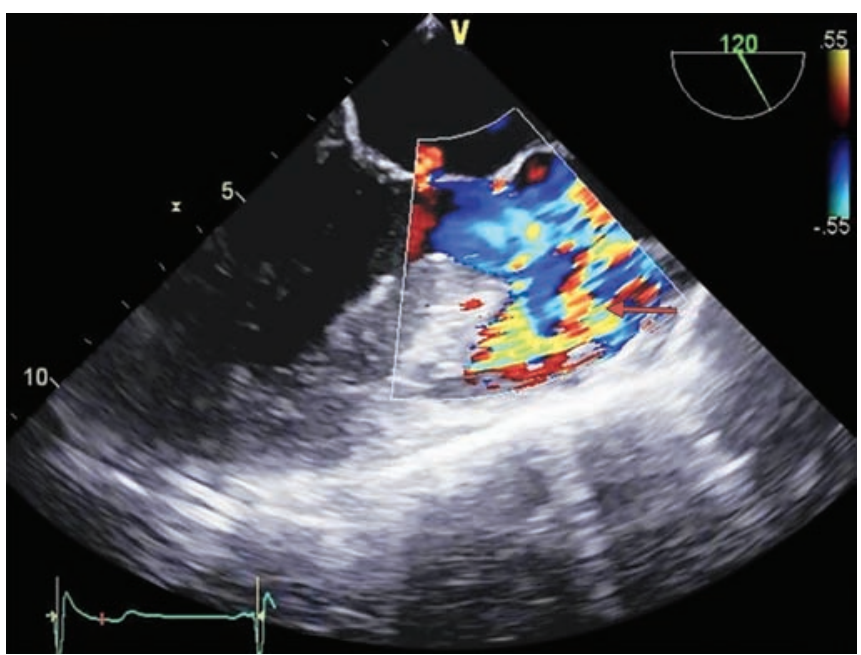

Fig. 6: Midesophageal aortic valve long axis view showing a late systolic flow (arrow) into right ventricular outflow tract due to ruptured sinus of valsalva aneurysm

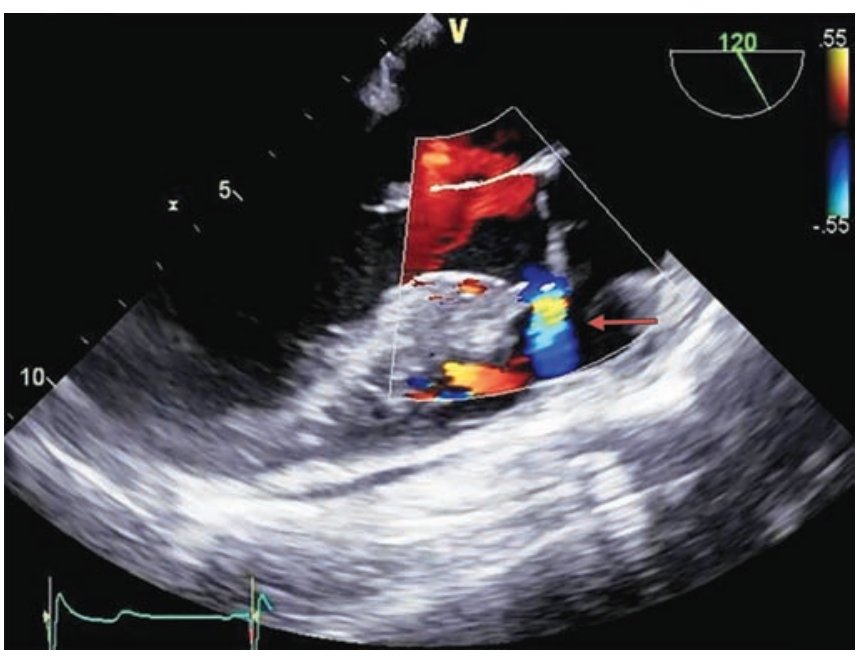

Fig. 5: Midesophageal aortic valve long axis view showing early systolic color flow (arrow) into right ventricular outflow tract due to a subpulmonic ventricular septal defect

increased our suspicion of patient having a VSD and RSOV. Rupture sinus of valsalva was responsible for acute clinical deterioration of the patient. There was no subaortic membrane or left ventricular outflow tract obstruction.

In our case, RSOV was associated with a subpulmonic VSD. Dong et a ${ }^{6}$ in a retrospective analysis $(n=67)$ found that subarterial VSD was most commonly associated (56\%) with a ruptured SOV aneurysm that involves RVOT. Echocardiography is the investigation of choice which can show characteristic windsock deformity and abnormal color flow can be seen on color Doppler application. The treatment of choice is surgery, which has 10 year survival rate of $>90 \%$ with closure of aneurysm and VSD. ${ }^{7}$ Percutaneous intervention with an Amplatzer Duct Occluder has also been found to be successful in selective cases and has been utilized in Europe. ${ }^{8}$

This case emphasizes the importance of routine TTE and TEE inside the operating room to look for new unanticipated findings. Mishra et $\mathrm{al}^{9}$ in their review found that routine intraoperative transthoracic and transesophageal echocardiography by cardiac anesthesiologist lead to new findings in around $5.5 \%$ of pediatric cardiac surgeries and change in surgical plan.

\section{REFERENCES}

1. Chu SH, Hung CR, How SS, et al. Ruptured aneurysms of the sinus of valsalva in oriental patients. J Thorac Cardiovasc Surg 1990;99(2):288-298

2. Takach TJ, Reul GJ, Duncan JM, et al. Sinus of valsalva aneurysm or fistula: management and outcome. Ann Thorac Surg 1999;68(5):1573-1577.

3. Fishbein MC, Obma R, Roberts WC. Unruptured sinus of valsalva aneurysm. Am J Cardiol 1975;35(6):918-922.

4. Ott DA. Aneurysm of the sinus of valsalva. Semin Thorac Cardiovasc Surg Pediatr Card Surg Annu 2006;19(1):165-176. 
5. Edwards JE, Burchell HB. Specimen exhibiting the essential lesion in aneurysm of the aortic sinus. Proc Staff Meet Mayo Clin 1956;31(14):407-412.

6. Dong C, Wu QY, Tang Y. Ruptured sinus of valsalva aneurysm: a Beijing experience. Ann Thorac Surg 2002;74(5): 1621-1624.

7. Van Son JA, Danielson GK, Schaff HV, et al. Long-term outcome of surgical repair of ruptured sinus of valsalva aneurysm. Circulation 1994;90(5):II20-29.
8. Kerkar PG, Lanjewar CP, Mishra N, et al. Transcatheter closure of ruptured sinus of valsalva aneurysm using the Amplatzer duct occluder: immediate results and mid-term follow-up. Eur Heart J 2010;31(23):2881-2887.

9. Mishra A, Madhavan JS, Ghuman BPS, et al. Impact and cost effectiveness of routine intraoperative transthoracic and transesophageal echocardiography on surgical decision making in pediatric cardiac surgery. J Periop Echo 2014;2(1): 3-9. 